\title{
UK Renal Registry 16th Annual Report: Appendix I Acronyms and Abbreviations used in the Report
}

\author{
ACE (inhibitor) \\ AKI \\ ANZDATA \\ APD \\ ADPKD \\ APKD \\ ATTOM \\ AV \\ AVF \\ AVG \\ BAPN \\ BCG \\ BCP \\ BMD \\ BMI \\ BP \\ BSI \\ BTS \\ $\mathrm{Ca}$ \\ $\mathrm{CAB}$ \\ CABG \\ CAPD \\ CCL \\ CCPD \\ CDI \\ Chol \\ $\mathrm{CHr}$ \\ CI \\ CK \\ CKD \\ CKD-EPI \\ CK-MB \\ COPD \\ CRF \\ CRF \\ CRP \\ CVVH \\ CXR \\ DBP \\ DCCT \\ $\mathrm{DH}$ \\ Angiotensin converting enzyme (inhibitor) \\ Acute kidney injury \\ Australia and New Zealand Dialysis and Transplant Registry \\ Automated peritoneal dialysis \\ Autosomal dominant polycystic kidney disease \\ Adult polycystic kidney disease \\ Access to transplant and transplant outcome measures \\ Arteriovenous \\ Arteriovenous fistula \\ Arteriovenous graft \\ British Association of Paediatric Nephrology \\ Bromocresol green \\ Bromocresol purple \\ Bone mineral disease \\ Body mass index \\ Blood pressure \\ Blood stream infection \\ British Transplant Society \\ Calcium \\ Clinical Affairs Board (Renal Association) \\ Coronary artery bypass grafting \\ Continuous ambulatory peritoneal dialysis \\ Clinical Computing Limited \\ Cycling peritoneal dialysis \\ Clostridium difficile infection \\ Cholesterol \\ Target reticulocyte $\mathrm{Hb}$ content \\ Confidence interval \\ Creatine kinase \\ Chronic kidney disease \\ Chronic kidney disease epidemiology collaboration \\ Creatine kinase isoenzyme MB \\ Chronic obstructive pulmonary disease \\ Chronic renal failure \\ Calculated HLA antibody reaction frequency \\ C-reactive protein \\ Continuous veno-venous haemofiltration \\ Chest x-ray \\ Diastolic blood pressure \\ Diabetes Control and Complications Trial \\ Department of Health
}

\section{KARGER}

Fax +4161306 1234

E-Mail karger@karger.com www.karger.com
(C) 2014 S. Karger AG, Basel

1160-2110/14/1254-0357\$38.00/0

Accessible online at: www.karger.com/nec
UK Renal Registry, Southmead Hospital, Southmead Road, Bristol, BS10 5NB, UK

Email: renalregistry@renalregistry.nhs.uk 


\begin{tabular}{|c|c|}
\hline DM & Diabetes mellitus \\
\hline $\mathrm{E} \& \mathrm{~W}$ & England and Wales \\
\hline EBPG & European Best Practice Guidelines \\
\hline ECG & Electrocardiogram \\
\hline EDTA & European Dialysis and Transplant Association \\
\hline $\mathrm{EF}$ & Error factor \\
\hline $\mathrm{E}_{\mathrm{i}}$ & Expected cases in area $\mathrm{i}$ \\
\hline ECD & Extended Criteria Donor \\
\hline EDTA & European Dialysis and Transplant Association \\
\hline $\mathrm{eKt} / \mathrm{V}$ & Equilibrated $\mathrm{Kt} / \mathrm{V}$ \\
\hline EPO & Erythropoietin \\
\hline ERA & European Renal Association \\
\hline ESRF & End stage renal failure \\
\hline EWNI & England, Wales and Northern Ireland \\
\hline Ferr & Ferritin \\
\hline FEV1 & Forced expiratory volume in 1 second \\
\hline FVC & Forced vital capacity \\
\hline GFR & Glomerular filtration rate \\
\hline $\mathrm{GH}$ & Growth hormone \\
\hline GN & Glomerulonephritis \\
\hline HA & Health Authority \\
\hline $\mathrm{HB}$ & Health board \\
\hline $\mathrm{Hb}$ & Haemoglobin \\
\hline HbAlc & Glycated Haemoglobin \\
\hline $\mathrm{HR}$ & Hazard ratio \\
\hline HRC & Hypochromic red blood cells \\
\hline $\mathrm{Ht}$ & Height \\
\hline ICU & Intensive care unit \\
\hline IDMS & Isotope dilution mass spectrometry \\
\hline IDOPPS & International Dialysis Outcomes and Practice Patterns Study \\
\hline IFCC & International Federation of Clinical Chemistry \& Laboratory Medicine \\
\hline IHD & Ischaemic heart disease \\
\hline IMD & Index of Multiple Deprivation \\
\hline IOTF & International Obesity Taskforce \\
\hline IPD & Intermittent peritoneal dialysis \\
\hline IQR & Inter-quartile range \\
\hline ISPD & International Society for Peritoneal Dialysis \\
\hline IT & Information technology \\
\hline IU & International units \\
\hline IV & Intra venous \\
\hline KDIGO & Kidney Disease: Improving Global Outcomes \\
\hline KDOQI & Kidney Disease Outcomes Quality Initiative \\
\hline $\mathrm{KM}$ & Kaplan Meier \\
\hline $\mathrm{Kt} / \mathrm{V}$ & $\begin{array}{l}\text { Ratio between the product of urea clearance }(\mathrm{K}, \mathrm{in} \mathrm{ml} / \mathrm{min}) \text { and dialysis session duration ( } \mathrm{t} \text {, in minutes) divided } \\
\text { by the volume of distribution of urea in the body }(\mathrm{V}, \text { in } \mathrm{ml})\end{array}$ \\
\hline
\end{tabular}




\begin{tabular}{|c|c|}
\hline LA & Local Authority \\
\hline LCL & Lower confidence limit \\
\hline LDL & Low-density lipoprotein \\
\hline LTFU & Lost to follow-up \\
\hline $\mathrm{M}: \mathrm{F}$ & Male:Female \\
\hline MAP & Mean arterial blood pressure \\
\hline MDRD & Modification of diet in renal disease \\
\hline MI & Myocardial infarction \\
\hline MMF & Mycophenolate mofetil \\
\hline MRSA & Methicillin resistant Staphylococcal aureus \\
\hline MSSA & Methicillin sensitive Staphylococcal aureus \\
\hline$N$ & Number \\
\hline $\mathrm{N}$ Ireland & Northern Ireland \\
\hline $\mathrm{NE}$ & North East \\
\hline NEQAS & UK National External Quality Assessment Scheme \\
\hline NHBPEP & National high blood pressure education programme \\
\hline NHS & National Health Service \\
\hline NHS BT & National Health Service Blood and Transplant \\
\hline NI & Northern Ireland \\
\hline NICE & National Institute for Health and Clinical Excellence \\
\hline NISRA & Northern Ireland Statistic and Research Agency \\
\hline $\mathrm{NMO}$ & Non-mixed origin \\
\hline NRS & National Records of Scotland \\
\hline NSF & National service framework \\
\hline NTC & Non-tunnelled dialysis catheter \\
\hline NTL & Non-tunnelled line \\
\hline NW & North West \\
\hline $\mathrm{O} / \mathrm{E}$ & Observed/expected \\
\hline ODT & Organ Donation and Transplantation (a Directorate of NHS Blood and transplant) \\
\hline $\mathrm{O}_{\mathrm{i}}$ & Observed cases in area i \\
\hline ONS & Office for National Statistics \\
\hline OR & Odds ratio \\
\hline PAS & Patient Administration System \\
\hline PCT & Primary Care Trust \\
\hline $\mathrm{PD}$ & Peritoneal dialysis \\
\hline PHE & Public Health England \\
\hline Phos & Phosphate \\
\hline PIAG & Patient Information Advisory Group \\
\hline PKD & Polycystic kidney disease \\
\hline PMARP & Per million age related population \\
\hline PMCP & Per million child population \\
\hline PMP & Per million population \\
\hline $\mathrm{PP}$ & Pulse pressure \\
\hline PRD & Primary renal disease \\
\hline PTH & Parathyroid hormone \\
\hline PUV & Posterior urethral valves \\
\hline PVD & Peripheral vascular disease \\
\hline QOF & Quality and Outcomes Framework \\
\hline QUEST & Quality European Studies \\
\hline RA & Renal Association \\
\hline $\mathrm{rhGH}$ & Recombinant human growth hormone \\
\hline RI & Royal Infirmary \\
\hline RNSF & Renal National Service Framework (or NSF) \\
\hline $\mathrm{RR}$ & Relative risk \\
\hline RRDSS & Renal Registry data set specification \\
\hline RRT & Renal replacement therapy \\
\hline RVD & Renovascular disease \\
\hline SAR & Standardised acceptance ratio $(=\mathrm{O} / \mathrm{E})$ \\
\hline SAS & Statistical Analysis System \\
\hline SBP & Systolic blood pressure \\
\hline SD & Standard deviation \\
\hline
\end{tabular}




$\begin{array}{ll}\text { SES } & \text { Socio-economic status } \\ \text { SHA } & \text { Strategic health authority } \\ \text { SHARP } & \text { Study of Heart and Renal Protection } \\ \text { SI } & \text { System International (units) } \\ \text { SMR } & \text { Standardised mortality ratios } \\ \text { spKt/V } & \text { Single pool Kt/V } \\ \text { SPR } & \text { Standardised prevalence ratio (=O/E) } \\ \text { SR } & \text { Standardised ratio (used to cover either SAR or SPR) } \\ \text { SUS } & \text { Secondary uses service } \\ \text { SW } & \text { South West } \\ \text { TC } & \text { Tunnelled dialysis catheter } \\ \text { TL } & \text { Tunnelled line } \\ \text { TSAT } & \text { Transferrin saturation } \\ \text { TWL } & \text { Transplant waiting list } \\ \text { Tx } & \text { Transplant } \\ \text { UCL } & \text { Upper confidence limit } \\ \text { UK } & \text { United Kingdom } \\ \text { UKRR } & \text { UK Renal Registry } \\ \text { UKT } & \text { UK Transplant (now ODT) } \\ \text { URR } & \text { Urea reduction ratio } \\ \text { US } & \text { United States } \\ \text { USA } & \text { United States of America } \\ \text { USRDS } & \text { United States Renal Data System } \\ \text { WHO } & \text { World health organization } \\ \text { Wt } & \text { Weight }\end{array}$

